\title{
DECOMPOSITION OF DIRICHLET FORMS ASSOCIATED TO UNBOUNDED DIRICHLET OPERATORS
}

\author{
Chul Ki Ko
}

\begin{abstract}
In [8], the author decomposed the Dirichlet form associated to a bounded generator $G$ of a weakly*-continuous, completely positive, KMS-symmetric Markovian semigroup on a von Neumann algebra $\mathcal{M}$. The aim of this paper is to extend $G$ to the unbounded generator using the bimodule structure and derivations.
\end{abstract}

\section{Introduction}

Noncommutative Dirichlet forms and Markovian semigroups on $C^{*}$-algebras or von Neumann algebras, pioneered by Albeverio and Hoegh-Krohn [2] in the tracial case and later extensively developed to the nontracial situations by Cipriani [4], were well understood at an analytic level [7, 11] and many constructions and applications were made $[5,7,9]$.

Many works were established on the structure of the generators of Markovian semigroups on von Neumann algebras. In [1], the authors showed that the generator of a symmetric conservative quantum dynamical semigroup with norm-bounded generator on a von Neumann algebra equipped with a faithful semi-finite trace is expressed as a sum of square of self-adjoint derivations. Cipriani and Sauvageot analyzed the structure of a tracially symmetric Dirichlet form on $C^{*}$-algebras or von Neumann algebras, in terms of a closable derivation taking values in a Hilbert space with a bimodule structure [6]. In [10], for a general Lindblad type generator $G$ of a KMS-symmetric quantum Markovian semigroup on a von Neumann algebra $\mathcal{M}$ acting on a Hilbert space $\mathcal{H}$, the author gave the sufficient condition so that the operator $H$ induced by $G$ via the symmetric embedding of $\mathcal{M}$ into $\mathcal{H}$ is symmetric and the type of a Dirichlet operator given in [9]. Recently, in [8], the Dirichlet form associated to a bounded generator $G$ of a weakly*-continuous, completely positive, KMS-symmetric Markovian semigroup on a von Neumann algebra $\mathcal{M}$ was decomposed.

Received May 30, 2008; Revised September 25, 2008.

2000 Mathematics Subject Classification. 46L55, 46L57, 47D07.

Key words and phrases. KMS-symmetric Markovian semigroups, Dirichlet forms, Dirichlet operators, derivations. 
The aim of this paper is to extend the generator $G$ studied in [8] to the unbounded operator. We study a weakly*-continuous, completely positive, KMS-symmetric Markovian semigroup $\left\{S_{t}\right\}_{t \geq 0}, S_{t}=e^{-t G}$ with an unbounded generator $G$ on a von Neumann algebra $\mathcal{M}$ acting on a separable Hilbert space $\mathcal{H}$. Modifying the bimodule structure used in [6], we construct a vector valued derivation on the algebra $\mathcal{M}$. Applying this derivation and the method used in [8], we decompose the Dirichlet form associated to the operator induced by $G$ via the symmetric embedding into an integral of forms whose associated generators are the divergence of derivations.

We organize the paper as follows: In Section 2, for a weakly*-continuous, completely positive, KMS-symmetric Markovian semigroup with unbounded generator on the von Neumann algebra, we give the associated Dirichlet form in the sense of Cipriani [4], and state the main result. Section 3 is devoted to the proofs of Proposition 2.1, Theorem 2.2 and Theorem 2.3. Using the similar method used in [6] and [8] (and also Section 1 of [11]), we construct a bimodule structure and a derivation, and decompose the associated Dirichlet form.

\section{Preliminaries and the main result}

In this section, we give the Dirichlet form in the sense of Cipriani [4] associated to a weakly*-continuous, completely positive, KMS-symmetric Markovian semigroup with unbounded generator on a von Neumann algebra, and state the main result.

Let $L(\mathfrak{h})$ be the von Neumann algebra of all bounded linear operators on a separable Hilbert space $\mathfrak{h}$ and the faithful, normal, semi-finite trace on $L(\mathfrak{h})$ is denoted Tr. Let $L^{2}(\mathfrak{h})$ be the Hilbert-Schmidt class, its inner product is given by $\langle\xi, \eta\rangle=\operatorname{Tr}\left(\xi^{*} \eta\right)$. Consider two maps

$$
\begin{aligned}
& \pi_{L}: L(\mathfrak{h}) \rightarrow B\left(L^{2}(\mathfrak{h})\right), \quad \pi_{L}(a)=L_{a}, \\
& \pi_{R}: L(\mathfrak{h}) \rightarrow B\left(L^{2}(\mathfrak{h})\right), \quad \pi_{R}(a)=R_{a},
\end{aligned}
$$

where $L_{a}$ and $R_{a}$ are the left and right multiplication operators on $L^{2}(\mathfrak{h})$, $L_{a}(\xi)=a \xi$ and $R_{a}(\xi)=\xi a, \xi \in L^{2}(\mathfrak{h})$, respectively. Put $\mathcal{M}=\pi_{L}(L(\mathfrak{h})), \mathcal{H}=$ $L^{2}(\mathfrak{h})$ and let $\mathcal{M}_{+}$be the set of positive elements in $\mathcal{M}$. Then the commutant $\mathcal{M}^{\prime}$ of $\mathcal{M}$ is $\pi_{R}(L(\mathfrak{h}))$.

Let $\rho$ be a (fixed) strictly positive density matrix, i.e., a strictly positive trace class operator on $\mathfrak{h}$ with $\operatorname{Tr}(\rho)=1$. Let $\omega$ be a faithful normal state on $\mathcal{M}$ defined by

$$
\omega\left(L_{a}\right)=\operatorname{Tr}(\rho a)=\left\langle\xi_{0}, a \xi_{0}\right\rangle, \quad a \in L(\mathfrak{h}),
$$

where $\xi_{0}=\rho^{1 / 2} \in \mathcal{H}$. Since $\rho$ is a strictly positive operator, $\xi_{0}$ is a cyclic and separating vector for $\mathcal{M}$. The associated modular operator $\Delta$ and modular automorphism group $\left\{\sigma_{t}\right\}_{t \in \mathbb{R}}$ are given by

$$
\begin{aligned}
& \Delta^{1 / 2} \xi=\rho^{1 / 2} \xi \rho^{-1 / 2}, \quad \xi \in L(\mathfrak{h}) \rho^{1 / 2}=L(\mathfrak{h}) \xi_{0}, \\
& \sigma_{t}\left(L_{a}\right)=\Delta^{i t} L_{a} \Delta^{-i t}=L_{\rho^{i t} a \rho^{-i t}}, \quad a \in L(\mathfrak{h}) .
\end{aligned}
$$


The element $a \in L(\mathfrak{h})$ may be identified as the left multiplicative operator $L_{a} \in \mathcal{M}$ on $\mathcal{H}$. We write $a:=L_{a}, \Delta^{i t} a \Delta^{-i t}:=\Delta^{i t} L_{a} \Delta^{-i t}$. Let $\mathcal{M}_{0}$ be the *-subalgebra of the $\sigma_{t}$-entire analytic elements in $\mathcal{M}[3]$. The state $\omega$ in $(2.1)$ satisfies the $\sigma$-KMS condition, $\omega(a b)=\omega\left(b \sigma_{-i}(a)\right), a, b \in \mathcal{M}_{0}$ and $\sigma$-invariant, for all $t \in \mathbb{R} \omega\left(\sigma_{t}(a)\right)=\omega(a), a \in \mathcal{M}$.

The closed convex cone $\mathcal{P}$ in $\mathcal{H}$ of nonnegative Hilbert-Schmidt operators is self-dual in the sense that $\{\xi \in \mathcal{H}:\langle\xi, \eta\rangle \geq 0, \forall \eta \in \mathcal{P}\}$. It is the closure of the set $\left\{\Delta^{1 / 4} a^{*} a \xi_{0}=\rho^{1 / 4} a^{*} a \rho^{1 / 4}: a \in \mathcal{M}_{0}\right\}$. The associated modular conjugation $J$ is an adjoint map on $\mathcal{H}, J \xi=\xi^{*}$ for $\xi \in \mathcal{H}$. $(\mathcal{M}, \mathcal{H}, \mathcal{P}, J)$ is a standard form for $L(\mathfrak{h})$.

The map $j: \mathcal{M} \rightarrow \mathcal{M}^{\prime}$ is the anti-linear $*$-isomorphism defined by $j(a)=$ $J a J, a \in \mathcal{M}$. The Hilbert space $\mathcal{H}$ is the complexification of the real subspace $\mathcal{H}^{J}:=\{\xi \in \mathcal{H}:\langle\xi, \eta\rangle \in \mathbb{R}, \forall \eta \in \mathcal{P}\}, \mathcal{H}=\mathcal{H}^{J} \oplus i \mathcal{H}^{J}$. For any two elements $\xi, \eta \in \mathcal{H}^{J}, \xi \leq \eta$ means $\eta-\xi \in \mathcal{P}$, thus $\mathcal{H}^{J}$ has an ordered structure. The order interval $I=\left\{\xi \in \mathcal{H}^{J}: 0 \leq \xi \leq \xi_{0}\right\}$, denoted by $\left[0, \xi_{0}\right]$, is a closed convex subset of $\mathcal{H}$. We denote by $\xi_{I}$ the nearest point projection of $\xi \in \mathcal{H}^{J}$ onto $I=\left[0, \xi_{0}\right]$. For the details we refer [4].

From now on we consider a (fixed) weakly*-continuous semigroup $\left\{S_{t}\right\}_{t \geq 0}$, $S_{t}=e^{-t G}$ on $\mathcal{M}$ with (unbounded) generator $G$ satisfying

$$
\begin{aligned}
& S_{t}(\mathbf{1})=\mathbf{1} \text { and } 0 \leq S_{t}(a) \leq\|a\| \mathbf{1}, \quad \forall a \in \mathcal{M}_{+} \text {(Markovian), } \\
& \omega\left(S_{t}(a) \sigma_{-i / 2}(b)\right)=\omega\left(\sigma_{i / 2}(a) S_{t}(b)\right), \quad \forall a, b \in \mathcal{M}_{0} \text { (KMS-symmetric) }
\end{aligned}
$$

for all $t \geq 0$ and

$$
\sum_{j, k=1}^{n} b_{j}^{*} S_{t}\left(a_{j}^{*} a_{k}\right) b_{k} \geq 0, \forall a_{j}, b_{j} \in \mathcal{M}, j=1, \ldots, n \text { (completely positive) }
$$

for all $t \geq 0$ and $n \in \mathbb{N}$. $S_{t}(\mathbf{1})=\mathbf{1}$ for all $t \geq 0$ implies $G(\mathbf{1})=0$, and the KMS-symmetric condition is written as

$$
\omega\left(G(a) \sigma_{-i / 2}(b)\right)=\omega\left(\sigma_{i / 2}(a) G(b)\right), \quad \forall a, b \in D(G) \cap \mathcal{M}_{0} .
$$

For the weakly*-continuous, completely positive, KMS-symmetric Markovian semigroup $\left\{S_{t}\right\}_{t \geq 0}$, define the semigroup $\left\{T_{t}\right\}_{t \geq 0}$ on $\mathcal{H}$ with generator $H$, $T_{t}=e^{-t H}$ by

$$
T_{t} \Delta^{1 / 4} a \xi_{0}=\Delta^{1 / 4} S_{t}(a) \xi_{0}, \forall a \in \mathcal{M}_{0},
$$

and the quadratic form $\mathcal{E}$ by

$$
\mathcal{E}\left(\Delta^{1 / 4} a \xi_{0}, \Delta^{1 / 4} b \xi_{0}\right)=\left\langle\Delta^{1 / 4} a \xi_{0}, H \Delta^{1 / 4} b \xi_{0}\right\rangle, \quad \forall a, b \in D(G) \cap \mathcal{M}_{0} .
$$

The closure of the quadratic form $\mathcal{E}$ is denoted by $(\mathcal{E}, D(\mathcal{E}))$ again. Then $\left\{T_{t}\right\}_{t>0}$ is a strongly continuous, symmetric, contraction semigroup on $\mathcal{H}$ and the generator $H$ is symmetric, positive on $\mathcal{H}$. Also the form $(\mathcal{E}, D(\mathcal{E}))$ satisfies

for any $\xi, \eta \in D(\mathcal{E}), \quad J \xi, J \eta \in D(\mathcal{E})$ and $\mathcal{E}(J \xi, J \eta)=\mathcal{E}(\eta, \xi)$ (J-real), and for any $\xi \in D(\mathcal{E}) \cap \mathcal{H}^{J}, \xi_{I} \in D(\mathcal{E})$ and $\mathcal{E}\left(\xi_{I}, \xi_{I}\right)$ (sub-Markovian). 
See Lemma 2.2, Theorem 2.11 and Theorem 4.11 of [4]. (2.4) implies

$$
H \Delta^{1 / 4} a \xi_{0}=\Delta^{1 / 4} G(a) \xi_{0}, \forall a \in D(G) \cap \mathcal{M}_{0} .
$$

In this case, $H$ and $(\mathcal{E}, D(\mathcal{E}))$ are called a Dirichlet operator and a Dirichlet form, respectively.

Assume that $B_{0}$ is a weakly*-dense subalgebra of $D(G)$ satisfying

(i) $\mathbf{1} \in B_{0} \subset \mathcal{M}_{0}$ and $G\left(B_{0}\right) \subset B_{0}$,

(ii) for any $a \in B_{0}, \quad \sigma_{t+i s}(a) \in B_{0}, \forall t, s \in \mathbb{R}$,

(iii) for any $a \in B_{0} \cap \mathcal{M}_{+}, a^{1 / 2} \in B_{0}$.

The above condition (iii) corresponds to one of sufficient conditions in order that the derivation on $\mathrm{C}^{*}$-algebra is dissipative (Proposition 3.2 .22 of [3]). When the density matrix $\rho$ is expressed as $\rho=\sum_{n=0}^{\infty} \lambda_{n}\left\langle\xi_{n}, \cdot\right\rangle \xi_{n}, \lambda_{n}>0$, where $\left\{\xi_{n}\right\}_{n=0}^{\infty}$ is an orthonormal basis of $\mathfrak{h}$, the class spanned by the elements expressed as the sum of the identity and finite rank operators with respect to the orthonormal basis satisfies the assumption (ii) and (iii).

Now we state our results, and their proofs are given in Section 3. We first introduce a Hilbert space $\mathcal{H}_{0}$ deduced from the algebraic tensor product space $B_{0} \otimes B_{0} \xi_{0}$ (Lemma 3.1) and construct a $B_{0}-B_{0}$ bimodule structure on $\mathcal{H}_{0}$.

Proposition 2.1. There exists a $B_{0}-B_{0}$ bimodule structure on $\mathcal{H}_{0}$ by

$$
\begin{aligned}
& a\left(b \otimes c \xi_{0}\right)=a b \otimes c \xi_{0}-a \otimes b c \xi_{0}, \\
& \left(b \otimes c \xi_{0}\right) a=b \otimes c a \xi_{0}
\end{aligned}
$$

for any $a, b, c \in B_{0}$.

Let $\partial$ be a map defined by

$$
\partial: B_{0} \rightarrow \mathcal{H}_{0}, \partial(a)=a \otimes \xi_{0} .
$$

By Proposition 2.1, $a \partial(b)=\partial(a b)-a \otimes b \xi_{0}$ and $\partial(a) b=a \otimes b \xi_{0}$ for all $a, b \in B_{0}$.

Theorem 2.2. (a) $\partial$ defined as in (2.7) is an $\mathcal{H}_{0}$-valued $B_{0}-B_{0}$ bimodule derivation:

$$
\partial(a b)=\partial(a) b+a \partial(b), a, b \in B_{0} .
$$

(b) There exists an antilinear isometric involution $\mathcal{J}$ of $\mathcal{H}_{0}$ satisfying

$$
\mathcal{J}(\partial(a))=\partial\left(\sigma_{-i / 2}\left(a^{*}\right)\right), a \in B_{0}
$$

and

$$
\mathcal{J}(a \xi b)=\sigma_{-i / 2}\left(b^{*}\right) \mathcal{J}(\xi) \sigma_{-i / 2}\left(a^{*}\right), a, b \in B_{0}, \xi \in \mathcal{H}_{0} .
$$

Now we state the main result of the paper and its proof is given in Section 3. The inner product and the induced norm of the Hilbert space $\mathcal{H}_{0}$ will be given in (3.5) and (3.6) respectively. The following is the main result of the paper:

Theorem 2.3. The Dirichlet form $(\mathcal{E}, D(\mathcal{E}))$ defined as in $(2.5)$ is expressed by

$$
\mathcal{E}\left(a \xi_{0}, a \xi_{0}\right)=\int\left\|\partial\left(\sigma_{t}(a)\right)\right\|_{\mathcal{H}_{0}}^{2} f_{0}(t) d t, \quad a \in B_{0}
$$


where the function $f_{0}: \mathbb{R} \rightarrow \mathbb{R}$ is given by

$$
f_{0}(t)=2\left(e^{2 \pi t}+e^{-2 \pi t}\right)^{-1}
$$

Remark 2.4. In case $\omega$ in (2.1) is a tracial state, the similar structure of bimodule in Proposition 2.1 and bimodule derivation in Theorem 2.2 have been introduced in [6], and the Dirichlet form $(\mathcal{E}, D(\mathcal{E}))$ has been decomposed. For a nontracial state and a bounded generator $G$, the decomposition of the Dirichlet form $(\mathcal{E}, D(\mathcal{E}))$ was established in $[8]$.

\section{Proof of the main result}

In this section, we produce the proofs of Proposition 2.1, Theorems 2.2 and 2.3. Using the similar method used in [6] and [8], we decompose the Dirichlet form $(\mathcal{E}, D(\mathcal{E}))$ in the sense of Cipriani [4] associated to a weakly*-continuous, completely positive, KMS-symmetric Makovian semigroup $\left\{S_{t}\right\}_{t \geq 0}$ with the unbounded generator $G$ on $\mathcal{M}$. See also Section 1 of [11].

For each $\varepsilon>0$, the resolvent maps defined by

$$
R_{\varepsilon}:=(\mathbf{1}+\varepsilon G)^{-1}=\int_{0}^{\infty} e^{-t} S_{\varepsilon t} d t
$$

are bounded, completely positive, normal contractions on $\mathcal{M}$, and the operators $G_{\varepsilon}:=G(\mathbf{1}+\varepsilon G)^{-1}=\varepsilon^{-1}\left(\mathbf{1}-R_{\varepsilon}\right), \varepsilon>0$, are bounded, positive preserving on $\mathcal{M}$. Then there exist a Hilbert space $\mathcal{H}_{\varepsilon}$, a $(*$-homomorphism) representation $\pi_{\varepsilon}$ of $\mathcal{M}$ into $L\left(\mathcal{H}_{\varepsilon}\right)$ and a linear operator $W_{\varepsilon}$ of $\mathcal{H}$ into $\mathcal{H}_{\varepsilon}$ with norm less than 1 , such that $R_{\varepsilon}(a)=W_{\varepsilon}^{*} \pi_{\varepsilon}(a) W_{\varepsilon}, a \in \mathcal{M}$.

Lemma 3.1. (a) For any $n \in \mathbb{N}, \varepsilon>0$ and $a_{1}, \ldots, a_{n} \in \mathcal{M}$, the $n \times n$ matrix $\left[\Lambda_{\varepsilon}\left(a_{j}^{*}, a_{k}\right)\right]_{j, k=1, \ldots, n}$ is positive, where $\Lambda_{\varepsilon}\left(a^{*}, b\right)=G_{\varepsilon}\left(a^{*}\right) b+a^{*} G_{\varepsilon}(b)-$ $G_{\varepsilon}\left(a^{*} b\right), a, b \in \mathcal{M}$.

(b) For any $a, b, c, d \in B_{0}$, the limit $\lim _{\varepsilon \downarrow 0} \omega\left(b^{*} \Lambda_{\varepsilon}\left(a^{*}, c\right) d\right)$ exists and equals

$$
\begin{aligned}
\lim _{\varepsilon \downarrow 0} \omega\left(b^{*} \Lambda_{\varepsilon}\left(a^{*}, c\right) d\right)= & \mathcal{E}\left(\sigma_{-i / 4}(a) \xi_{0}, \sigma_{i / 4}(c d) \sigma_{-3 i / 4}\left(b^{*}\right) \xi_{0}\right) \\
& +\mathcal{E}\left(\sigma_{i / 4}(a b) \sigma_{-3 i / 4}\left(d^{*}\right) \xi_{0}, \sigma_{-i / 4}(c) \xi_{0}\right) \\
& -\mathcal{E}\left(\sigma_{i / 4}(b) \sigma_{-3 i / 4}\left(d^{*}\right) \xi_{0}, \sigma_{-i / 4}\left(a^{*} c\right) \xi_{0}\right) .
\end{aligned}
$$

Proof. (a) Let $a_{j}, b_{j} \in \mathcal{M}, j=1, \ldots, n$. Using $G_{\varepsilon}=\varepsilon^{-1}\left(\mathbf{1}-R_{\varepsilon}\right)$ and $R_{\varepsilon}(a)=$ $W_{\varepsilon}^{*} \pi_{\varepsilon}(a) W_{\varepsilon}, \varepsilon>0$ in the above, we have

$$
\begin{aligned}
& \Lambda_{\varepsilon}\left(a_{j}^{*}, a_{k}\right) \\
= & \varepsilon^{-1}\left(a_{j}^{*} a_{k}-R_{\varepsilon}\left(a_{j}^{*}\right) a_{k}-a_{j}^{*} R_{\varepsilon}\left(a_{k}\right)+R_{\varepsilon}\left(a_{j}^{*} a_{k}\right)\right) \\
(3.3)= & \varepsilon^{-1}\left[\left(W_{\varepsilon} a_{j}-\pi_{\varepsilon}\left(a_{j}\right) W_{\varepsilon}\right)^{*}\left(W_{\varepsilon} a_{k}-\pi_{\varepsilon}\left(a_{k}\right) W_{\varepsilon}\right)+a_{j}^{*}\left(1-W_{\varepsilon}^{*} W_{\varepsilon}\right) a_{k}\right] .
\end{aligned}
$$


Thus we get from (3.3) that

$$
\begin{aligned}
& \sum_{j, k=1}^{n} b_{j}^{*} \Lambda_{\varepsilon}\left(a_{j}^{*}, a_{k}\right) b_{k} \\
= & \varepsilon^{-1}\left(\sum_{j=1}^{n}\left(W_{\varepsilon} a_{j}-\pi_{\varepsilon}\left(a_{j}\right) W_{\varepsilon}\right) b_{j}\right)^{*}\left(\sum_{j=1}^{n}\left(W_{\varepsilon} a_{j}-\pi_{\varepsilon}\left(a_{j}\right) W_{\varepsilon}\right) b_{j}\right) \\
& +\varepsilon^{-1}\left(\sum_{j=1}^{n}\left(1-W_{\varepsilon}^{*} W_{\varepsilon}\right)^{1 / 2} a_{j} b_{j}\right)^{*}\left(\sum_{j=1}^{n}\left(1-W_{\varepsilon}^{*} W_{\varepsilon}\right)^{1 / 2} a_{j} b_{j}\right) \\
\geq & 0,
\end{aligned}
$$

which implies (a).

(b) By the definition of $G_{\varepsilon}$, the limit $\lim _{\varepsilon \downarrow 0} \omega\left(b^{*} \Lambda_{\varepsilon}\left(a^{*}, c\right) d\right)$ exists and equals $\omega\left(b^{*} \Lambda\left(a^{*}, c\right) d\right)$, where $\Lambda\left(a^{*}, c\right)=G\left(a^{*}\right) c+a^{*} G(c)-G\left(a^{*} c\right)$. Applying the definition of the Dirichlet form $(\mathcal{E}, D(\mathcal{E}))$ and KMS-symmetric condition of $G$, we easily obtain the relation $(3.2)$.

Due to Lemma 3.1, we can define a Hilbert space $\mathcal{H}_{0}$ deduced from the algebraic tensor product space $B_{0} \otimes B_{0} \xi_{0}$ with respect to the inner product $(\cdot, \cdot)$ defined by

$$
\begin{aligned}
\left(a \otimes b \xi_{0}, c \otimes d \xi_{0}\right)= & \mathcal{E}\left(\sigma_{-i / 4}(a) \xi_{0}, \sigma_{i / 4}(c d) \sigma_{-3 i / 4}\left(b^{*}\right) \xi_{0}\right) \\
& +\mathcal{E}\left(\sigma_{i / 4}(a b) \sigma_{-3 i / 4}\left(d^{*}\right) \xi_{0}, \sigma_{-i / 4}(c) \xi_{0}\right) \\
& -\mathcal{E}\left(\sigma_{i / 4}(b) \sigma_{-3 i / 4}\left(d^{*}\right) \xi_{0}, \sigma_{-i / 4}\left(a^{*} c\right) \xi_{0}\right)
\end{aligned}
$$

for any $a, b, c, d \in B_{0}$, and the associated norm $\|\cdot\|_{\mathcal{H}_{0}}$

$$
\begin{aligned}
\left\|a \otimes b \xi_{0}\right\|_{\mathcal{H}_{0}}^{2}= & \mathcal{E}\left(\sigma_{-i / 4}(a) \xi_{0}, \sigma_{i / 4}(a b) \sigma_{-3 i / 4}\left(b^{*}\right) \xi_{0}\right) \\
& +\mathcal{E}\left(\sigma_{i / 4}(a b) \sigma_{-3 i / 4}\left(b^{*}\right) \xi_{0}, \sigma_{-i / 4}(a) \xi_{0}\right) \\
& -\mathcal{E}\left(\sigma_{i / 4}(b) \sigma_{-3 i / 4}\left(b^{*}\right) \xi_{0}, \sigma_{-i / 4}\left(a^{*} a\right) \xi_{0}\right)
\end{aligned}
$$

for any $a, b \in B_{0}$.

By $(2.3)$ and $G(\mathbf{1})=0$, we have $\omega\left(G\left(a^{*} a\right)\right)=0$ for any $a \in B_{0}$, and so

$$
\begin{aligned}
E(a) & :=\left\|a \otimes \xi_{0}\right\|_{\mathcal{H}_{0}}^{2} \\
& =\omega\left(G\left(a^{*}\right) a+a^{*} G(a)\right) .
\end{aligned}
$$

Lemma 3.2. For any $n \in \mathbb{N}, a, a_{1}, \ldots, a_{n}, b_{1}, \ldots, b_{n} \in B_{0}$, two inequalities

$$
\begin{aligned}
& \left\|\sum_{j=1}^{n} a_{j} \otimes b_{j} a \xi_{0}\right\|_{\mathcal{H}_{0}} \leq\left\|\sigma_{-i / 2}\left(a^{*}\right)\right\|\left\|\sum_{j=1}^{n} a_{j} \otimes b_{j} \xi_{0}\right\|_{\mathcal{H}_{0}}, \\
& \left\|\sum_{j=1}^{n}\left(a a_{j} \otimes b_{j} \xi_{0}-a \otimes a_{j} b_{j} \xi_{0}\right)\right\|_{\mathcal{H}_{0}} \leq\|a\|\left\|\sum_{j=1}^{n} a_{j} \otimes b_{j} \xi_{0}\right\|_{\mathcal{H}_{0}}
\end{aligned}
$$

hold. Moreover, $\left\|a \otimes b \xi_{0}\right\|_{\mathcal{H}_{0}} \leq\left\|\sigma_{-i / 2}\left(b^{*}\right)\right\| \sqrt{E(a)}, a, b \in B_{0}$. 
Proof. Notice that by $\sigma$-KMS condition, the inequality

$$
\omega\left(b^{*} a^{*} a b\right) \leq\left\|\sigma_{-i / 2}\left(b^{*}\right)\right\|^{2} \omega\left(a^{*} a\right), \quad a, b \in B_{0}
$$

holds.

Let $a, a_{j}, b_{j} \in B_{0}, j=1, \ldots, n$. Due to Lemma 3.1 (a), for any $\varepsilon>0$, $\sum_{j, k=1}^{n} b_{j}^{*} \Lambda_{\varepsilon}\left(a_{j}^{*}, a_{k}\right) b_{k}$ is positive. We get from the assumption of $B_{0}$ and (3.10) that

$$
\begin{aligned}
\left\|\sum_{j=1}^{n} a_{j} \otimes b_{j} a \xi_{0}\right\|_{\mathcal{H}_{0}}^{2} & =\lim _{\varepsilon \downarrow 0} \omega\left(a^{*}\left(\sum_{j, k=1}^{n} b_{j}^{*} \Lambda_{\varepsilon}\left(a_{j}^{*}, a_{k}\right) b_{k}\right) a\right) \\
& \leq\left\|\sigma_{-i / 2}\left(a^{*}\right)\right\|^{2} \lim _{\varepsilon \downarrow 0} \omega\left(\sum_{j, k=1}^{n} b_{j}^{*} \Lambda_{\varepsilon}\left(a_{j}^{*}, a_{k}\right) b_{k}\right) \\
& =\left\|\sigma_{-i / 2}\left(a^{*}\right)\right\|^{2}\left\|\sum_{j=1}^{n} a_{j} \otimes b_{j} \xi_{0}\right\|_{\mathcal{H}_{0}}^{2},
\end{aligned}
$$

which implies (3.8).

Next, to produce the inequality (3.9), we estimate

$$
\begin{aligned}
&\left\|\sum_{j=1}^{n}\left(a a_{j} \otimes b_{j} \xi_{0}-a \otimes a_{j} b_{j} \xi_{0}\right)\right\|_{\mathcal{H}_{0}}^{2} \\
&= \sum_{j, k=1}^{n}\left(a a_{j} \otimes b_{j} \xi_{0}-a \otimes a_{j} b_{j} \xi_{0}, a a_{k} \otimes b_{k} \xi_{0}-a \otimes a_{k} b_{k} \xi_{0}\right) \\
&= \sum_{j, k=1}^{n} \lim _{\varepsilon \downarrow 0} \omega\left(b _ { j } ^ { * } \left[G_{\varepsilon}\left(a_{j}^{*} a^{*} a\right) a_{k}+a_{j}^{*} G_{\varepsilon}\left(a^{*} a a_{k}\right)\right.\right. \\
&\left.\left.-a_{j}^{*} G_{\varepsilon}\left(a^{*} a\right) a_{k}-G_{\varepsilon}\left(a_{j}^{*} a^{*} a a_{k}\right)\right] b_{k}\right) .
\end{aligned}
$$

By the similar calculation used in Lemma 3.1, for $\varepsilon>0$ we have

$$
\begin{aligned}
& \sum_{j, k=1}^{n} b_{j}^{*}\left[G_{\varepsilon}\left(a_{j}^{*} a^{*} a\right) a_{k}+a_{j}^{*} G_{\varepsilon}\left(a^{*} a a_{k}\right)-a_{j}^{*} G_{\varepsilon}\left(a^{*} a\right) a_{k}-G_{\varepsilon}\left(a_{j}^{*} a^{*} a a_{k}\right)\right] b_{k} \\
= & \varepsilon^{-1} \sum_{j, k=1}^{n} b_{j}^{*}\left(W_{\varepsilon} a_{j}-\pi_{\varepsilon}\left(a_{j}\right) W_{\varepsilon}\right)^{*} \pi_{\varepsilon}\left(a^{*} a\right)\left(W_{\varepsilon} a_{k}-\pi_{\varepsilon}\left(a_{k}\right) W_{\varepsilon}\right) b_{k} \\
\leq & \varepsilon^{-1}\|a\|^{2} \sum_{j, k=1}^{n} b_{j}^{*}\left(W_{\varepsilon} a_{j}-\pi_{\varepsilon}\left(a_{j}\right) W_{\varepsilon}\right)^{*}\left(W_{\varepsilon} a_{k}-\pi_{\varepsilon}\left(a_{k}\right) W_{\varepsilon}\right) b_{k} \\
= & \|a\|^{2} \sum_{j, k=1}^{n} b_{j}^{*}\left[G_{\varepsilon}\left(a_{j}^{*}\right) a_{k}+a_{j}^{*} G_{\varepsilon}\left(a_{k}\right)-G_{\varepsilon}\left(a_{j}^{*} a_{k}\right)\right] b_{k} \\
& -\varepsilon^{-1}\|a\|^{2} \sum_{j, k=1}^{n} b_{j}^{*} a_{j}^{*}\left(1-W_{\varepsilon}^{*} W_{\varepsilon}\right) a_{k} b_{k} .
\end{aligned}
$$


Here we have used $\left\|\pi_{\varepsilon}\left(a^{*} a\right)\right\| \leq\|a\|^{2}$ in the inequality, and (3.4) in second equality. Substituting (3.12) into (3.11), and using $W_{\varepsilon}^{*} W_{\varepsilon} \leq \mathbf{1}$, we get

$$
\begin{aligned}
& \left\|\sum_{j=1}^{n} a a_{j} \otimes b_{j} \xi_{0}-a \otimes \sum_{j=1}^{n} a_{j} b_{j} \xi_{0}\right\|_{\mathcal{H}_{0}}^{2} \\
\leq & \|a\|^{2} \lim _{\varepsilon \downarrow 0} \omega\left(\sum_{j, k=1}^{n} b_{j}^{*}\left[G_{\varepsilon}\left(a_{j}^{*}\right) a_{k}+a_{j}^{*} G_{\varepsilon}\left(a_{k}\right)-G_{\varepsilon}\left(a_{j}^{*} a_{k}\right)\right] b_{k}\right) \\
= & \|a\|^{2}\left\|\sum_{j=1}^{n} a_{j} \otimes b_{j} \xi_{0}\right\|_{\mathcal{H}_{0}}^{2} .
\end{aligned}
$$

Thus (b) holds. Finally the rest follows from (3.8) and $\left\|a \otimes \xi_{0}\right\|=\sqrt{E(a)}$.

Due to Lemma 3.2, we construct structures of a right $B_{0}$-module and a left $B_{0}$-module on $\mathcal{H}_{0}$.

Proposition 3.3. There exist structures of a right $B_{0}$-module on $\mathcal{H}_{0}$ by

$$
\left(\sum_{j=1}^{n} a_{j} \otimes b_{j} \xi_{0}\right) a=\sum_{j=1}^{n} a_{j} \otimes b_{j} a \xi_{0}
$$

and a left $B_{0}$-module on $\mathcal{H}_{0}$ by

$$
a\left(\sum_{j=1}^{n} a_{j} \otimes b_{j} \xi_{0}\right)=\sum_{j=1}^{n}\left(a a_{j} \otimes b_{j} \xi_{0}-a \otimes a_{j} b_{j} \xi_{0}\right)
$$

for all $n \in \mathbb{N}, a, a_{j}, b_{j} \in B_{0}, j=1, \ldots, n$. The involutions

$$
\left(a \otimes b \xi_{0},\left(c \otimes d \xi_{0}\right) e\right)=\left(\left(a \otimes b \xi_{0}\right) \sigma_{-i}\left(e^{*}\right), c \otimes d \xi_{0}\right)
$$

and

$$
\left(e\left(a \otimes b \xi_{0}\right), c \otimes d \xi_{0}\right)=\left(a \otimes b \xi_{0}, e^{*}\left(c \otimes d \xi_{0}\right)\right)
$$

hold for any $a, b, c, d, e \in B_{0}$.

Proof. By Lemma 3.2 and continuity, we have the right multiplication by $\left(\sum_{j=1}^{n} a_{j} \otimes b_{j} \xi_{0}\right) a=\sum_{j=1}^{n} a_{j} \otimes b_{j} a \xi_{0}$ and the left multiplication by $a\left(\sum_{j=1}^{n} a_{j} \otimes\right.$ $\left.b_{j} \xi_{0}\right)=\sum_{j=1}^{n}\left(a a_{j} \otimes b_{j} \xi_{0}-a \otimes a_{j} b_{j} \xi_{0}\right)$ for all $n, a, a_{j}, b_{j} \in B_{0}, j=1, \ldots, n$.

By the direct calculation we have the involutions: for any $a, b, c, d, e \in B_{0}$

$$
\begin{aligned}
\left(a \otimes b \xi_{0},\left(c \otimes d \xi_{0}\right) e\right) & =\lim _{\varepsilon \downarrow 0} \omega\left(b^{*} \Lambda_{\varepsilon}\left(a^{*}, c\right) d e\right) \\
& =\lim _{\varepsilon \downarrow 0} \omega\left(\sigma_{i}(e) b^{*} \Lambda_{\varepsilon}\left(a^{*}, c\right) d\right) \\
& =\left(a \otimes b \sigma_{-i}\left(e^{*}\right) \xi_{0}, c \otimes d \xi_{0}\right) \\
& =\left(\left(a \otimes b \xi_{0}\right) \sigma_{-i}\left(e^{*}\right), c \otimes d \xi_{0}\right)
\end{aligned}
$$


and

$$
\begin{aligned}
\left(e\left(a \otimes b \xi_{0}\right), c \otimes d \xi_{0}\right)= & \left(e a \otimes b \xi_{0}-e \otimes a b \xi_{0}, c \otimes d \xi_{0}\right) \\
= & \lim _{\varepsilon \downarrow 0} \omega\left(b^{*}\left[G_{\varepsilon}\left(a^{*} e^{*}\right) c+a^{*} e^{*} G_{\varepsilon}(c)-G_{\varepsilon}\left(a^{*} e^{*} c\right)\right] d\right. \\
& \left.\quad-b^{*} a^{*}\left[G_{\varepsilon}\left(e^{*}\right) c+e^{*} G_{\varepsilon}(c)-G\left(e^{*} c\right)\right] d\right) \\
= & \lim _{\varepsilon \downarrow 0} \omega\left(b^{*}\left[G_{\varepsilon}\left(a^{*}\right) e^{*} c+a^{*} G_{\varepsilon}\left(e^{*} c\right)-G_{\varepsilon}\left(a^{*} e^{*} c\right)\right] d\right. \\
& \left.\quad-b^{*}\left[G_{\varepsilon}\left(a^{*}\right) e^{*}+a^{*} G_{\varepsilon}\left(e^{*}\right)-G\left(a^{*} e^{*}\right)\right] c d\right) \\
= & \left(a \otimes b \xi_{0}, e^{*} c \otimes d \xi_{0}\right)-\left(a \otimes b \xi_{0}, e^{*} \otimes c d \xi_{0}\right) \\
= & \left(a \otimes b \xi_{0}, e^{*}\left(c \otimes d \xi_{0}\right)\right) .
\end{aligned}
$$

The proof is completed.

Proof of Proposition 2.1. The proof is directly obtained from Proposition 3.3.

Recall that $\partial(a)=a \otimes \xi_{0}$ and $\partial(a) b=a \otimes b \xi_{0}$ for all $a, b \in B_{0}$.

Lemma 3.4. For any $a, b, c, d \in B_{0}$, the following relations hold:

(a) $(\partial(a), c \partial(b))=\left(\partial\left(\sigma_{-i / 2}\left(b^{*}\right)\right), \partial\left(\sigma_{-i / 2}\left(a^{*}\right)\right) \sigma_{-i / 2}(c)\right)$,

(b) $(\partial(a), \partial(b))=\left(\partial\left(\sigma_{-i / 2}\left(b^{*}\right)\right), \partial\left(\sigma_{-i / 2}\left(a^{*}\right)\right)\right)$,

(c) $(\partial(a), c \partial(b) d)=\left(\sigma_{-i / 2}\left(d^{*}\right) \partial\left(\sigma_{-i / 2}\left(b^{*}\right)\right) \sigma_{-i / 2}\left(c^{*}\right), \partial\left(\sigma_{-i / 2}\left(a^{*}\right)\right)\right)$.

Proof. (a) We rewrite (2.3) as

$$
\omega(G(a) b)=\omega\left(\sigma_{i / 2}(a) G\left(\sigma_{i / 2}(b)\right)\right), \quad a, b \in B_{0} .
$$

Let $a, b, c \in B_{0}$. By $\omega\left(G\left(a^{*} c b\right)\right)=0$ and $\sigma$-KMS condition, one has

$$
\begin{aligned}
(\partial(a), c \partial(b)) & =\left(a \otimes \xi_{0}, c b \otimes \xi_{0}-c \otimes b \xi_{0}\right) \\
& =\omega\left(G\left(a^{*} c\right) b+a^{*} G(c b)-a^{*} G(c) b\right) \\
& =\omega\left(G\left(a^{*} c\right) b+G(c b) \sigma_{-i}\left(a^{*}\right)-G(c) b \sigma_{-i}\left(a^{*}\right)\right) .
\end{aligned}
$$

Applying (3.13) and $\sigma$-KMS condition to (3.14), we get

$$
\begin{aligned}
(\partial(a), c \partial(b))= & \omega\left(\sigma_{i / 2}\left(a^{*} c\right) G\left(\sigma_{i / 2}(b)\right)+\sigma_{i / 2}(c b) G\left(\sigma_{-i / 2}\left(a^{*}\right)\right)\right. \\
& \left.-\sigma_{i / 2}(c) G\left(\sigma_{i / 2}(b) \sigma_{-i / 2}\left(a^{*}\right)\right)\right) \\
= & \omega\left(G\left(\sigma_{i / 2}(b)\right) \sigma_{-i / 2}\left(a^{*} c\right)+\sigma_{i / 2}(b) G\left(\sigma_{-i / 2}\left(a^{*}\right)\right) \sigma_{-i / 2}(c)\right. \\
& \left.-G\left(\sigma_{i / 2}(b) \sigma_{-i / 2}\left(a^{*}\right)\right) \sigma_{-i / 2}(c)\right) \\
= & \left(\sigma_{-i / 2}\left(b^{*}\right) \otimes \xi_{0}, \sigma_{-i / 2}\left(a^{*}\right) \otimes \sigma_{-i / 2}(c) \xi_{0}\right) \\
= & \left(\partial\left(\sigma_{-i / 2}\left(b^{*}\right)\right), \partial\left(\sigma_{-i / 2}\left(a^{*}\right)\right) \sigma_{-i / 2}(c)\right) .
\end{aligned}
$$

(b) Putting $c=1$ in (a), we obtain the relation.

(c) Let $a, b, c, d \in B_{0}$. Notice that

$$
(\partial(a), c \partial(b) d)=(\partial(a), c \partial(b d))-(\partial(a), c b \partial(d)) .
$$


Applying (a) to the above, and using the involutions in Proposition 3.3, we have

$$
\begin{aligned}
(\partial(a), c \partial(b) d)= & \left(\partial\left(\sigma_{-i / 2}\left(d^{*} b^{*}\right)\right), \partial\left(\sigma_{-i / 2}\left(a^{*}\right)\right) \sigma_{-i / 2}(c)\right) \\
& -\left(\partial\left(\sigma_{-i / 2}\left(d^{*}\right)\right), \partial\left(\sigma_{-i / 2}\left(a^{*}\right)\right) \sigma_{-i / 2}(c b)\right) \\
= & \left(\partial\left(\sigma_{-i / 2}\left(d^{*} b^{*}\right)\right) \sigma_{-i / 2}\left(c^{*}\right), \partial\left(\sigma_{-i / 2}\left(a^{*}\right)\right)\right) \\
& -\left(\partial\left(\sigma_{-i / 2}\left(d^{*}\right)\right) \sigma_{-i / 2}\left(b^{*} c^{*}\right), \partial\left(\sigma_{-i / 2}\left(a^{*}\right)\right)\right) \\
= & \left(\sigma_{-i / 2}\left(d^{*}\right) \partial\left(\sigma_{-i / 2}\left(b^{*}\right)\right) \sigma_{-i / 2}\left(c^{*}\right), \partial\left(\sigma_{-i / 2}\left(a^{*}\right)\right) .\right.
\end{aligned}
$$

The proof is completed.

Proof of Theorem 2.2. (a) The definition of $\partial$ and the bimodule structure directly produce (a).

(b) Notice that $B_{0} \otimes B_{0} \xi_{0}$ is dense in $\mathcal{H}_{0}$. By Lemma 3.4, there exists an antilinear isometric involution $\mathcal{J}$ of $\mathcal{H}_{0}$ satisfying $\mathcal{J}(\partial(a))=\partial\left(\sigma_{-i / 2}\left(a^{*}\right)\right), a \in$ $B_{0}$ and $\mathcal{J}(a \xi b)=\sigma_{-i / 2}\left(b^{*}\right) \mathcal{J}(\xi) \sigma_{-i / 2}\left(a^{*}\right), a, b \in B_{0}, \xi \in \mathcal{H}_{0}$.

To characterize the structure of Dirichlet form $(\mathcal{E}, D(\mathcal{E}))$, we introduce a lemma.

Lemma 3.5. Let $C$ be a bounded operator on $\mathcal{H}$. For any $a, b \in \mathcal{M}_{0}$, the relation

$$
\begin{aligned}
& \int\left[\left\langle\Delta^{i t+1 / 4} a \xi_{0}, C \Delta^{i t-1 / 4} b \xi_{0}\right\rangle+\left\langle\Delta^{i t-1 / 4} a \xi_{0}, C \Delta^{i t+1 / 4} b \xi_{0}\right\rangle\right] f_{0}(t) d t \\
= & \left\langle a \xi_{0}, C b \xi_{0}\right\rangle
\end{aligned}
$$

holds, where $f_{0}(t)$ is defined as in (2.8).

Proof. See the proof of Proposition 4.1 of [8]. See also the proof of Lemma 3.1 of $[10]$.

Proof of Theorem 2.3. Let $a \in B_{0}$. By assumption (ii) of $B_{0}, \sigma_{t}(a) \in B_{0}$ for all $t \in \mathbb{R}$. Since $\Delta^{1 / 4}$ is closed and $G_{\varepsilon}(a) \xi_{0} \in D\left(\Delta^{1 / 4}\right)$ for any $\varepsilon>0$, we get from (2.4) that $\Delta^{1 / 4}(1+\varepsilon G)^{-1}(a) \xi_{0}=H_{\varepsilon} \Delta^{1 / 4} a \xi_{0}$, and so $\Delta^{1 / 4} G_{\varepsilon}(a) \xi_{0}=$ $H_{\varepsilon} \Delta^{1 / 4} a \xi_{0}$, where $H_{\varepsilon}=H(\mathbf{1}+\varepsilon H)^{-1}$.

By (3.7) and the dominated convergence theorem, we have

$$
\begin{aligned}
& \int\left\|\partial\left(\sigma_{t}(a)\right)\right\|_{\mathcal{H}_{0}}^{2} f_{0}(t) d t \\
= & \int \lim _{\varepsilon \downarrow 0} \omega\left(G_{\varepsilon}\left(\sigma_{t}\left(a^{*}\right)\right) \sigma_{t}(a)+\sigma_{t}\left(a^{*}\right) G_{\varepsilon}\left(\sigma_{t}(a)\right)\right) f_{0}(t) d t \\
= & \lim _{\varepsilon \downarrow 0} \int \omega\left(G_{\varepsilon}\left(\sigma_{t}\left(a^{*}\right)\right) \sigma_{t}(a)+\sigma_{t}\left(a^{*}\right) G_{\varepsilon}\left(\sigma_{t}(a)\right)\right) f_{0}(t) d t .
\end{aligned}
$$


Using the relation $\Delta^{1 / 4} G_{\varepsilon}(a) \xi_{0}=H_{\varepsilon} \Delta^{1 / 4} a \xi_{0}$ and $\Delta^{i t} \xi_{0}=\xi_{0}$ for all $t \in \mathbb{R}$, we have

$$
\begin{aligned}
& \int \omega\left(G_{\varepsilon}\left(\sigma_{t}\left(a^{*}\right)\right) \sigma_{t}(a)+\sigma_{t}\left(a^{*}\right) G_{\varepsilon}\left(\sigma_{t}(a)\right)\right) f_{0}(t) d t \\
= & \int\left[\left\langle G_{\varepsilon}\left(\sigma_{t}(a)\right) \xi_{0}, \sigma_{t}(a) \xi_{0}\right\rangle+\left\langle\sigma_{t}(a) \xi_{0}, G_{\varepsilon}\left(\sigma_{t}(a)\right) \xi_{0}\right\rangle\right] f_{0}(t) d t \\
= & \int\left[\left\langle\Delta^{i t+1 / 4} a \xi_{0}, H_{\varepsilon} \Delta^{i t-1 / 4} a \xi_{0}\right\rangle+\left\langle\Delta^{i t-1 / 4} a \xi_{0}, H_{\varepsilon} \Delta^{i t+1 / 4} a \xi_{0}\right\rangle\right] f_{0}(t) d t \\
= & \left\langle a \xi_{0}, H_{\varepsilon} a \xi_{0}\right\rangle .
\end{aligned}
$$

Since for any $\xi \in D(H) H_{\varepsilon} \xi \rightarrow H \xi$ as $\varepsilon \downarrow 0$, we get

$$
\begin{aligned}
\int\left\|\partial\left(\sigma_{t}(a)\right)\right\|_{\mathcal{H}_{0}}^{2} f_{0}(t) d t & =\left\langle a \xi_{0}, H a \xi_{0}\right\rangle \\
& =\mathcal{E}\left(a \xi_{0}, a \xi_{0}\right) .
\end{aligned}
$$

The proof is completed.

Acknowledgment. The author would like to thank the anonymous referee for suggestions made for the improvement of the paper.

\section{References}

[1] S. Albeverio and D. Goswami, A remark on the structure of symmetric quantum dynamical semigroups on von Neumann algebras, Infin. Dimens. Anal. Quantum Probab. Relat. Top. 5 (2002), no. 4, 571-579.

[2] S. Albeverio and R. Høegh-Krohn, Dirichlet forms and Markov semigroups on $C^{*}$ algebras, Comm. Math. Phys. 56 (1977), no. 2, 173-187.

[3] O. Bratteli and D. W. Robinson, Operator algebras and quantum-statistical mechanics. II, Equilibrium states. Models in quantum-statistical mechanics. Texts and Monographs in Physics. Springer-Verlag, New York-Berlin, 1981.

[4] F. Cipriani, Dirichlet forms and Markovian semigroups on standard forms of von Neumann algebras, J. Funct. Anal. 147 (1997), no. 2, 259-300.

[5] F. Cipriani, F. Fagnola, and J. M. Lindsay, Spectral analysis and Feller property for quantum Ornstein-Uhlenbeck semigroups, Comm. Math. Phys. 210 (2000), no. 1, 85105.

[6] F. Cipriani and J.-L. Sauvageot, Derivations as square roots of Dirichlet forms, J. Funct. Anal. 201 (2003), no. 1, 78-120.

[7] E. B. Davies and J. M. Lindsay, Noncommutative symmetric Markov semigroups, Math. Z. 210 (1992), no. 3, 379-411.

[8] C. K. Ko, Remarks on the decomposition of Dirichlet forms on standard forms of von Neumann algebras, J. Math. Phys. 48 (2007), no. 11, 113504, 11 pp.

[9] Y. M. Park, Construction of Dirichlet forms on standard forms of von Neumann algebras, Infin. Dimens. Anal. Quantum Probab. Relat. Top. 3 (2000), no. 1, 1-14.

[10] - Remarks on the structure of Dirichlet forms on standard forms of von Neumann algebras, Infin. Dimens. Anal. Quantum Probab. Relat. Top. 8 (2005), no. 2, 179-197.

[11] J.-L. Sauvageot, Tangent bimodule and locality for dissipative operators on $C^{*}$-algebras, Quantum probability and applications, IV (Rome, 1987), 322-338, Lecture Notes in Math., 1396, Springer, Berlin, 1989. 
University College

YONSEI UNIVERSITY

SEOUl 120-749, KoreA

E-mail address: kochulki@yonsei.ac.kr 